\title{
Formation of millisecond pulsars - NS initial mass and EOS constraints
}

\author{
Michał Bejger $^{1}$, Morgane Fortin ${ }^{1,2}$, Paweł Haensel $^{1}$ \\ and J. Leszek Zdunik ${ }^{1}$
}

\author{
${ }^{1}$ N. Copernicus Astronomical Center PAS, Bartycka 18, PL-00-716 Warsaw, Poland \\ email: bejger, haensel, jlz@camk.edu.pl \\ ${ }^{2}$ LUTH, UMR 8102 du CNRS, Observatoire de Paris, F-92195 Meudon Cedex, France \\ email: morgane.fortin@obspm.fr
}

\begin{abstract}
Recent measurement of a high millisecond pulsar mass (PSR J1614-2230, $1.97 \pm$ $\left.0.04 M_{\odot}\right)$ compared with the low mass of PSR J0751+1807 $\left(1.26 \pm 0.14 M_{\odot}\right)$ indicates a large span of masses of recycled pulsars and suggests a broad range of neutron stars masses at birth. We aim at reconstructing the pre-accretion masses for these pulsars while taking into account interaction of the magnetic field with a thin accretion disk, magnetic field decay and relativistic $2 \mathrm{D}$ solutions for stellar configurations for a set of equations of state. We briefly discuss the evolutionary scenarios leading to the formation of these neutron stars and study the influence of the equation of state.
\end{abstract}

Keywords. Stars: neutron, pulsars: individual, accretion disks, equation of state, magnetic fields

\section{Introduction}

The discovery of a $1.97 \pm 0.04 M_{\odot}$ rapidly-rotating neutron star (NS) (Demorest et al. 2010) motivates the theorists to understand its origin and composition. Population of millisecond pulsars contains also a low-mass PSR J0751+1807 $\left(1.26 \pm 0.14 M_{\odot}\right.$, Nice et al. 2008); a statistical analysis of the measured and estimated pulsar masses, in particular of the millisecond ones, was presented in (Zhang et al. 2011). Despite a striking difference in their masses, their spin periods and inferred magnetic fields are similar $(B \propto \sqrt{P \dot{P}}$, see Table 1). Both of them were spun-up by accretion from a binary companion. The evolution of such systems is typically composed of several stages: starting from the Zero Age Main Sequence (ZAMS), more massive primary (NS progenitor) enters the Red Giant (RG) phase and engulfs the secondary for a brief Common Envelope (CE) phase. Subsequently the primary, now as a helium star of a substantially lower mass, explodes as a core-collapse supernova (SN), forming the NS. Secondary star evolves from a ZAMS star into a RG and spins-up the NS via the Roche lobe overflow disk accretion. Depending on stellar masses, the resulting composition of the secondary, binary orbital periods and semi-major axes may be very different, which in turn may result in additional stages of evolution: Intermediate Mass X-ray Binary (IMXB) stage, connected with the Terminal Age Main Sequence instabilities in the case of PSR J1914-2230, or secondary evaporation in a Low Mass X-ray Binary (LMXB) - so-called Black Widow scenario - in the case of PSR J0751+1807 (see Table 2 for quantitative figures and De Loore \& Doom 1992, Tauris \& van den Heuvel 2006 and Tauris et al. 2011 for more details).

\section{Methods}

Hereunder we focus on the recycling/spin-up stage of the evolution leading to the formation of a millisecond pulsar. It is modeled by a sequence of stationary rotating 
Table 1. Selected parameters of millisecond pulsars PSR J1614-2230 and PSR J0751+1807 and their binary systems (orbital period $P_{\mathrm{b}}$, eccentricity $e$ and the companion mass $M_{\mathrm{WD}}$; mass measurement errors reflect $1-\sigma$ confidence levels).

\begin{tabular}{|c|c|c|c|c|c|c|}
\hline PSR & $M\left[M_{\odot}\right]$ & $P[\mathrm{~ms}]$ & $\dot{P}\left[10^{-21} s\right]$ & $P_{\mathrm{b}}[\mathrm{d}]$ & $e$ & $M_{\mathrm{WD}}\left[M_{\odot}\right]$ \\
\hline $\mathrm{J}$ & $1.97 \pm 0.04$ & 3.15 & 9.6 & $8.7 \mathrm{~d}$ & $1.3 \times 10^{-6}$ & $0.500 \pm 0.006(\mathrm{CO} W \mathrm{WD})$ \\
\hline $\mathrm{J} 0751+1807$ & $1.26 \pm 0.14$ & 3.48 & 7.2 & $6 \mathrm{~h}$ & $5.6 \times 10^{-5}$ & $0.12 \pm 0.02(\mathrm{He} W D)$ \\
\hline
\end{tabular}

configurations of increasing mass, calculated for a given equation of state (EOS). Rigidly rotating axisymmetric stellar configuration were obtained by means of the library for relativistic computations LORENE. Increase of the total stellar angular momentum $J$ is calculated by accounting for the transfer of the angular momentum from the thin accretion disk interacting with the NS magnetosphere:

$$
\frac{\mathrm{d} J}{\mathrm{~d} M_{\mathrm{b}}}=l_{0}-\frac{K_{B}}{\dot{M}},
$$

where an accreted (baryon) mass $\mathrm{d} M_{b}$ carries a specific angular momentum $l_{0}$ from the inner boundary of a disk; $\dot{M}$ is the accretion rate and $K_{B}$ denotes a magnetic (braking) torque. We employ the prescription of Kluźniak \& Rappaport (2007), improved by considering the relativistic effects (the innermost stable circular orbit, Bejger et al. 2011a) and using approximate, but accurate representation for orbital parameters of particles in the disk (Bejger et al. 2010). Gradual magnetic field decay, which is caused by the infalling accreted matter, is taken into account as well; we adopt the relation of Shibazaki et al. (1989) and Taam \& van den Heuvel (1986) that links the decrease of $\vec{B}$ with the amount of accreted matter only (actual evolution of the polar magnetic field of an accreting NS can be more complicated, see Zhang \& Kojima 2006 and references therein).

Following EOSs were used: APR by Akmal et al. (1998), DH by Douchin \& Haensel (2001) and BM by Bednarek et al. (2011). APR EOS (A18 + $\delta v+$ UIX $^{*}$ model) is a variational, non-relativistic many-body solution with relativistic corrections (maximal mass of a non-rotating star: $\left.M_{\max }^{\text {stat }}=2.19 M_{\odot}, R\left(M_{\max }^{\text {stat }}\right)=9.93 \mathrm{~km}\right)$. DH EOS is constructed using a non-relativistic energy density functional based on the SLy4 effective nuclear interaction, designed to describe both crust and core in an unified way $\left(M_{\max }^{\text {stat }}=2.05 M_{\odot}, R\left(M_{\max }^{\text {stat }}\right)=10.0 \mathrm{~km}\right)$. BM EOS is based on relativistic mean field model, with a non-linear Lagrangian that includes quartic terms in meson fields, and two additional hidden-strangeness mesons $\sigma^{*}$ and $\phi$ that couple to hyperons only $\left(M_{\max }^{\text {stat }}=2.03 M_{\odot}, R\left(M_{\max }^{\text {stat }}\right)=10.7 \mathrm{~km}\right)$.

Detailed description of methods and tests can be found in Bejger et al. (2011a).

Table 2. Evolutionary stages for PSR J1614-2230 and PSR J0751+1807 systems.

\begin{tabular}{ccc|c|cc|c|c}
\hline & & ZAMS & 1st RG & CE & SNII & 2nd RG & now \\
\hline J1614-2230 & primary & $25 \mathrm{M}_{\odot}$ & $5 \times 10^{6} \mathrm{y}$ & $7 \mathrm{M}_{\odot}$ & $1.9 \mathrm{M}_{\odot}$ & & $1.97 \mathrm{M}_{\odot}$ \\
& secondary & $4.5 \mathrm{M}_{\odot}$ & & $4.5 \mathrm{M}_{\odot}$ & $4.5 \mathrm{M}_{\odot}$ & $5 \times 10^{7} \mathrm{y}$ & $0.50 \mathrm{M}_{\odot}$ \\
& $P_{\text {orb }}$ & $1 \mathrm{y}$ & & $4 \mathrm{~d}$ & $2 \mathrm{~d}$ & & $8.7 \mathrm{~d}$ \\
\hline $\mathrm{J} 0751+1807$ & primary & $15 \mathrm{M}_{\odot}$ & $10^{7} \mathrm{y}$ & $5 \mathrm{M}_{\odot}$ & $1.1 \mathrm{M}_{\odot}$ & & $1.26 \mathrm{M}_{\odot}$ \\
& secondary & $1.6 \mathrm{M}_{\odot}$ & & $1.6 \mathrm{M}_{\odot}$ & $1.6 \mathrm{M}_{\odot}$ & $10^{9} \mathrm{y}$ & $0.12 \mathrm{M}_{\odot}$ \\
& $P_{\text {orb }}$ & $1 \mathrm{y}$ & & $1 \mathrm{~d}$ & $1.5 \mathrm{~d}$ & & $6 \mathrm{~h}$ \\
\hline
\end{tabular}




\section{Results}

The recycling process for PSR J1614-2230 and J0751+1807 is presented in Fig. 1 and 2 , respectively, and visualized in terms of accretion time $\tau$, the amount of accreted mass $M_{\text {acc }}$, and stellar spin frequency $f$. We assume constant accretion rate $\dot{M}$ during the recycling. The lifetime of an LMXB or IMXB system puts strong limits on the amount of accreted mass and $\dot{M}$. In the case of J1614-2230, such an evolutionary argument of Tauris et al. (2011) places a constraint on the accretion time $\tau: \tau \simeq 100 \mathrm{Myr}$, which results in a pre-accretion mass of this NS very close to the observed mass: $>1.9 M_{\odot}$, and a way to estimate $\dot{M}$ needed for the spin-up (see second term in Eq. 2.1; small $\tau$ requires large $\dot{M}$ close to the Eddington limit).

From Fig. 1 and 2 one can deduce the minimal amount of accreted mass needed to reach the observed $\simeq 300 \mathrm{~Hz}$ spin frequency: $\Delta M_{\min } \simeq 0.04 \div 0.05 M_{\odot}$ for $\tau<1 \mathrm{Gyr}$. Hence, $\dot{M}$ in such a case is

$$
\dot{M}=\frac{\Delta M_{\min }}{\tau} \simeq 10^{-9} M_{\odot} / \mathrm{yr} \tau_{50}^{-1},
$$

where $\tau_{50}=\tau / 50$ Myr. For PSR J0751+1807, our simulations indicate an initial mass of $\simeq 1.2 M_{\odot}$; its value is a function the accretion time $\tau, \dot{M}$ and initial magnetic field (see right panel in Fig. 2).

The influence of the dense-matter EOS can be summarized in terms of its stiffness: the stiffer the EOS, the larger the moment of inertia $I$ for a given mass $\left(I \sim M R^{2}\right)$; it requires therefore more accretion of angular momentum to spin-up a star to a given frequency in a given amount of time (see Eq. 2.1). While the DH and APR EOSs give very similar results for observed frequencies, the BM EOS differs from them since it must be much stiffer ( $I$ is $20 \%$ higher than for DH EOS) to sustain the softening by hyperons (see e.g., left panel on Fig. 2).
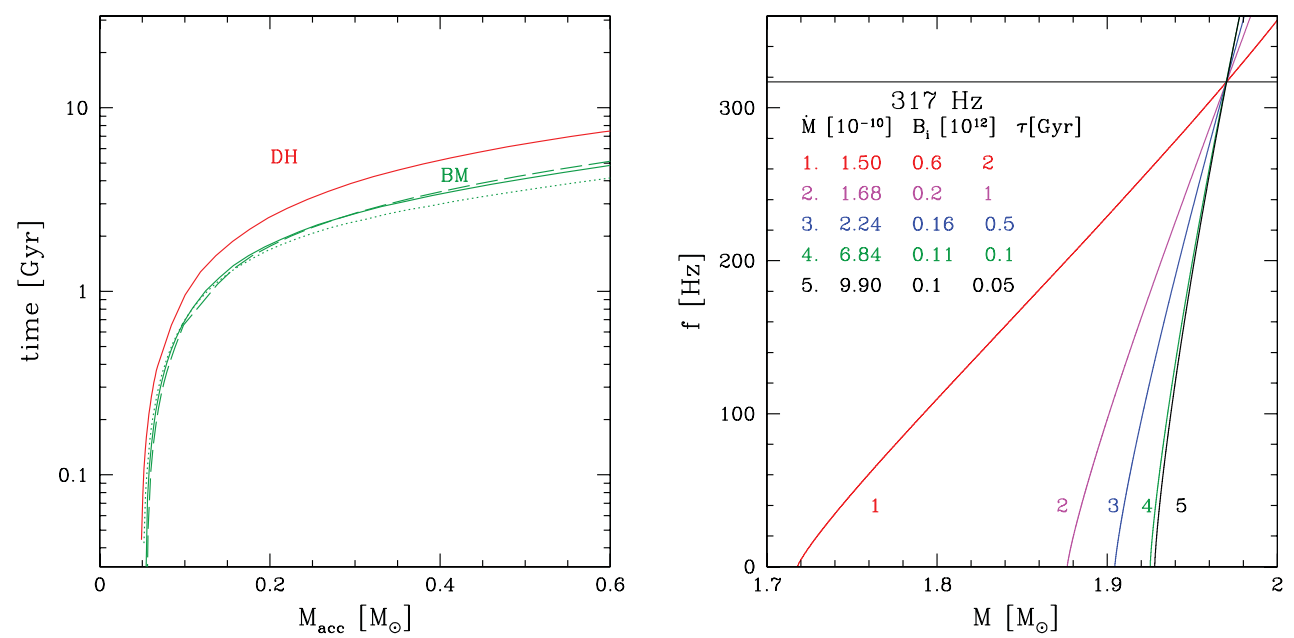

Figure 1. Currently most massive millisecond pulsar PSR J1614-2230. Left panel: Accreting time needed to spin-up the star to presently observed values as a function of accreted mass for $\mathrm{DH}$ and BM EOSs $\left(B_{i}=10^{12} \mathrm{G}\right.$, dotted and dashed lines correspond to lower and upper mass limit, 1.93 and $2.01 M_{\odot}$ ). Right panel: selected spin-up tracks parametrized by different initial masses, magnetic fields, accretion rates and times. Lines cross at the current values of the pulsar parameters (BM EOS). 

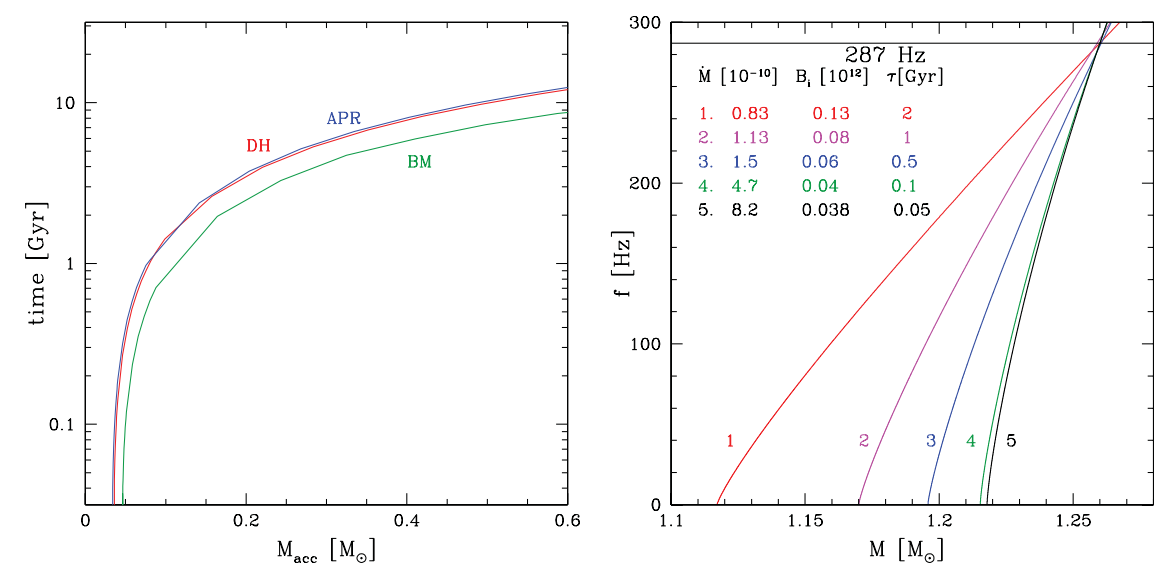

Figure 2. As in Figure 1, but for a low-mass millisecond pulsar PSR J0751+1807.

\section{Summary}

We present a versatile method of studying the formation and evolution of millisecond pulsars in the recycling phase. This prescription allows for testing the magnetic field decay and magnetic torque models, in the framework of a relativistic thin accretion disk spin-up scenario. Coupled with evolutionary data, the information relevant to the SN collapse simulations, spin and initial mass distribution of pulsar populations can be drawn from the timing data and mass measurements. We point out that from the densematter EOS point of view, both the low and the high end of the initial mass spectrum is very important, as both the minimal and the maximal initial NS mass contains the information about the composition of the matter and processes occurring at its birth. Moreover, the disk-magnetic field interaction in our model changes dramatically the character of the evolution of the interior and the spin of NS, as compared to the results where $\vec{B}$ is neglected (Bejger et al. 2011b).

Acknowledgements. This work was partially supported by the Polish MNiSW research grant no. 2011/01/B/ST9/04838.

\section{References}

Akmal, A., Pandharipande, V. R., \& Ravenhall, D. G. 1998, Phys. Rev. C, 58, 1804

Bednarek, I., et al. 2011, A\&GA, 543, A157

Bejger, M., Zdunik, J. L., \& Haensel, P. 2010, A\&A, 520, A16

Bejger, M., Fortin, M., Haensel, P., \& Zdunik, J. L. 2011, A\& A, 536, A87

Bejger, M., Haensel, P., Zdunik, J. L., \& Fortin, M. 2011, A\&A, 536, A92

Demorest, P. B., et al. 2010, Nature, 467, 1081

De Loore, C. W. H. \& Doom, C., 1992, Astrophysics and Space Science Library, 179

Douchin, F. \& Haensel, P. 2001, A\&A, 380, 151

Kluźniak, W. \& Rappaport, S. 2007, ApJ, 671, 1990

Nice, D. J., Stairs, I. H., \& Kasian, L. E. 2008, 40 Years of Pulsars: Millisecond Pulsars, Magnetars and More, 983, 453

Shibazaki, N., Murakami, T., Shaham, J., \& Nomoto, K. 1989, Nature, 342, 656

Taam, R. E. \& van den Heuvel, E. P. J., 1986, ApJ, 305, 235

Tauris, T. M. \& van den Heuvel, E. P. J. 2006, Compact stellar X-ray sources, Cambridge Astrophysics Series, No. 39, 623

Tauris, T. M., Langer, N., \& Kramer, M. 2011, MNRAS, 416, 2130

Zhang, C. M. \& Kojima, Y. 2006, MNRAS, 366, 137

Zhang, C. M., Wang, J., Zhao, Y. H., et al. 2011, A\& A, 527, A83 